\title{
Análisis de dificultades surgidas al resolver problemas de variación
}

\section{Analysis of arising difficulties when solving variation problems}

\author{
Claudia Barajas Arenas ${ }^{1}$ \\ Sandra Evely Parada Rico ${ }^{2}$ \\ Juan Gabriel Molina Zavaleta ${ }^{3}$
}

\begin{abstract}
Resumen: La reprobación en cursos de Cálculo Diferencial del primer ciclo universitario ha sido reportada internacionalmente (Artigue, 1995, Díaz, 2009) como un gran problema, asumiendo como una de las causas la falta de conocimiento en matemáticas que tienen los estudiantes graduados de la educación preuniversitaria. En este trabajo reportamos elementos de una investigación centrada en las dificultades que presentan estudiantes universitarios cuando resuelven problemas que implican situaciones de variación. Específicamente, se estudiaron dificultades asociadas al proceso matemático de elaboración, comparación y ejercitación de procedimientos (MEN, 1998). Inicialmente, analizamos una prueba diagnóstica de un curso de precálculo en el que participaron estudiantes de nuevo ingreso a la universidad. Se encontró que los estudiantes tienen dificultades para establecer relaciones y diferencias entre notaciones de números reales y decidir sobre su uso en una situación dada. Además, fueron evidentes las dificultades para emplear diferentes representaciones y reconocer el efecto de una representación u otra en la resolución de un problema. Estas dificultades se
\end{abstract}

Fecha de recepción: 1 de abril de 2017. Fecha de aceptación: 15 de octubre de 2018.

1 Grupo de Educación Matemática de la UIS Universidad Industrial de Santander, Colombia, claubaren@ gmail.com, orcid.org/0000-0003-3499-9484

2 Universidad Industrial de Santander, sanevepa@uis.edu.co,orcid.org/0000-0001-5468-0943

3 Instituto Politécnico Nacional, Programa de Matemática Educativa, Cicata-Legaria, jmolinaz@ipn.mx, orcid.org/0000-0001-6547-7131 
ilustran a partir del análisis de uno de los problemas que figuraron en la prueba y que se considera paradigmático. La identificación y caracterización de estas dificultades puede permitir a los profesores de cálculo tomar decisiones importantes sobre su planificación didáctica.

Palabras clave: dificultades, pensamiento variacional, procedimientos, resolución de problemas, variación.

\begin{abstract}
Failing in Differential Calculus courses of the first cycle in university has been reported internationally (Artigue, 1995, Diaz, 2009) as a big problem, assuming as one of the causes the lack of knowledge in mathematics that graduated high school students have. In this work we report elements of a research focused on the difficulties that first year university students face when they solve problems that involve variation situations. Specifically, difficulties associated to the mathematical process development, comparison and training of procedures (MEN, 1998). At first, we analyzed a diagnostic test of a pre-calculus course in which newly enrolled university students participated. It was found that students have difficulties to establish relationships and differences between different notations of real numbers and to decide on their use in a given situation. Further, the difficulties of using the representations and recognizing the effect of using one representation or another in the resolution of the problem were evident. The identification and characterization of these difficulties may allow to calculus teachers to take important decisions about their didactic planning.
\end{abstract}

Keywords: difficulties, variational thinking, procedures, solving problems, variation.

\title{
1. INTRODUCCIÓN
}

El Cálculo Diferencial implica resolver problemas de variación, acumulación y tendencia y sin embargo, el proceso de instrucción se ha venido enfocando en las técnicas algorítmicas, con lo cual se trivializa la resolución de problemas. Artigue (1995) señaló la dificultad en lograr que los estudiantes muestren una comprensión satisfactoria de los conceptos y procedimientos; situación que se ha constituido en una problemática en los primeros niveles universitarios; en 
Cálculo Diferencial, asignatura en el primer semestre del plan de estudios de 15 carreras profesionales de la Universidad Industrial de Santander (UIS), contexto en el que se desarrolló la investigación que fundamenta esta contribución. Los estudiantes de nuevo ingreso presentan dificultades significativas reflejadas en los índices de reprobación: en 2012 y 2013 se ofrecieron 40 cursos en promedio, cada uno de ellos con 40 estudiantes, la reprobación aproximada en cálculo diferencial fue de $49 \%$ y $64 \%$ respectivamente (Botello, 2013), situación preocupante para instancias académico-administrativas de dicha universidad.

Con el objetivo de reconocer elementos que inciden en la reprobación, se han desarrollado varios estudios sobre las dificultades que poseen los estudiantes al ingresar a esta universidad, entre ellos los de Abrate, Pochulu y Vargas (2006), Cadenas (2007). En estos estudios se da cuenta de diferentes carencias, dificultades y errores en los conocimientos matemáticos en alumnos del primer semestre universitario, evidenciando preconceptos deficientes sobre las nociones de cambio y variación. Considerando estos antecedentes, se decidió desarrollar un estudio sobre las concepciones de los estudiantes en torno a la variación. Para ello se diseñó y analizó una prueba diagnóstica que incluye problemas variacionales y que se ha venido implementando desde el año 2014 a los estudiantes de nuevo ingreso a los programas de ingenierías y ciencias en la UIS (Parada, 2012). La prueba está conformada por preguntas de selección múltiple con única respuesta alrededor de problemas de fenómenos variacionales, que requieren el uso del pensamiento variacional, de la reflexión sobre el cambio en los fenómenos involucrados, del reconocimiento cualitativo y cuantitativo del cambio en el sistema u objeto que se estudia, lo cual es en esencia lo que se hace en Cálculo Diferencial.

El análisis de respuestas a estas pruebas ha permitido caracterizar dificultades de los estudiantes, particularmente las asociadas a la resolución de problemas que requieren un pensamiento variacional, el cual se relaciona con diferentes tipos de procesos. Se considera que si el profesor de matemáticas reconoce estas dificultades, podría anticipar la forma en que conviene organizar didácticamente ciertos temas, así como elegir, diseñar o adaptar actividades matemáticas que permitan a los estudiantes superar dichas dificultades.

La caracterización de dificultades se hizo con base en la definición de pensamiento variacional y de diferentes tipos de procedimientos con los que se relaciona, como se detalla en la siguiente sección. 


\section{ASPECTOS TEÓRICOS}

\subsection{Pensamiento VARIACIONAL}

Los Lineamientos Curriculares de Matemáticas de Colombia, como un caso de la enseñanza superior en América Latina, proponen el estudio de la variación desde el pensamiento variacional, considerando que el currículo de matemáticas se centra en tres elementos: los contextos, los procesos matemáticos y, como eje central, el pensamiento matemático que es subdividido en cinco pensamientos articulados entre sí: el pensamiento numérico, métrico, aleatorio, espacial y variacional (MEN, 2006).

El pensamiento variacional es comprendido en este estudio como

un campo conceptual, que involucra conceptos y procedimientos interestructurados y vinculados que permitan analizar, organizar y modelar matemáticamente situaciones y problemas tanto de la actividad práctica del hombre, como de las ciencias y las propiamente matemáticas donde la variación se encuentre como sustrato de ellas. En esta forma se amplía la visión de la variación, por cuanto su estudio se inicia en el intento de cuantificar la variación por medio de las cantidades y las magnitudes (MEN, 1998, p. 72).

Cabe señalar que los procedimientos involucrados en este pensamiento son las actuaciones, destrezas, estrategias, métodos, técnicas, usos y aplicaciones diversas que un estudiante realiza para resolver problemas de manera cada vez más hábil e independiente (Barajas, 2015). Barringer, Pohlman y Robinson (2010) señalan que la comprensión de los procedimientos en matemáticas fomenta la comprensión de los conceptos matemáticos y la capacidad de resolver problemas.

El pensamiento variacional se desarrolla en estrecha relación con los otros tipos de pensamiento porque

la variación y el cambio, aunque se representan usualmente por medio de sistemas algebraicos y analíticos, requieren de conceptos y procedimientos relacionados con distintos sistemas numéricos (en particular, del sistema de los números reales, fundamentales en la construcción de las funciones de variable real), geométricos, de medidas y de datos y porque todos estos sistemas, a su vez, pueden presentarse en forma estática o en forma dinámica y variacional (MEN, 1998, p. 66). 
Se asume que el pensamiento variacional permite comprender los fenómenos de variación ya que tiene que ver con el reconocimiento, la percepción, identificación y caracterización de la variación y el cambio en diferentes contextos. Se reconocen tres ejes conceptuales asociados al pensamiento variacional que orientan el desarrollo del currículo de la educación básica y media: patrones y regularidades, procesos algebraicos y análisis de funciones (Seduca, 2005).

\subsection{PROCEDIMIENTOS Y SU TAXONOMÍA}

Algunos autores (Rico, 1995; MEN, 1998; Bronzina, Chemello, y Agrasar, 2009) distinguen varios grupos de procedimiento según el campo de las matemáticas escolares donde operan. Particularmente, Rico (1995) habla de destrezas, consideradas como formas de actuación o ejecución de tareas. Distingue cinco destrezas de los procedimientos: aritméticas, métricas, geométricas, gráficas y de representación. Dichas destrezas consisten en "transformar una expresión simbólica desde una forma dada hasta otra forma, y para ello hay que ejecutar una secuencia de reglas sobre manipulación de símbolos" (Rico, 1995, p.15).

Los procedimientos permiten tener una mirada a las ideas, imágenes, representaciones y a los contextos que los estudiantes evocan en la resolución de distintos problemas (Valdivé y Garbin, 2013); éstos pueden ser entendidos desde la taxonomía de procedimientos:

- Procedimientos aritméticos. Relacionados con el uso adecuado del número y la estructura del sistema de numeración decimal; de las operaciones en diversos contextos; de sus propiedades y de las relaciones entre ellas.

- Procedimientos geométricos. Asociados a utilizar atributos medibles y propiedades de figuras y objetos 2D y 3D y su ubicación en el plano o el espacio; las nociones de horizontalidad, verticalidad, paralelismo y perpendicularidad; los diseños y construcciones utilizando representaciones de cuerpos y figuras geométricas; las representaciones verbales y gráficas de recorridos y el reconocimiento de ángulos y polígonos, su clasificación y propiedades en la resolución de problemas.

- Procedimientos métricos. Implican la capacidad para construir conceptos de cada magnitud, procesos de conservación, unidades de medida, estimación de magnitudes y de rangos, seleccionar y usar unidades de medida y de patrones, sistemas monetarios y el sistema métrico decimal. 
- Procedimientos analíticos. Relativos al uso del "Álgebra”, "Cálculo Diferencial e Integral"; con el reconocimiento de regularidades y patrones, la caracterización de la variación, la identificación de variables, la descripción de fenómenos de cambio y dependencia en contextos aritméticos y geométricos.

Dentro de cada procedimiento se consideran, a su vez, procedimientos algorítmicos y no algorítmicos, ya que cada uno de éstos responde a un conjunto de directrices para trabajar con los problemas de matemáticas.

La taxonomía de procedimientos permite categorizar las dificultades emergentes de la resolución de problemas, a partir de cuatro habilidades que están relacionadas con cada tipo de procedimiento, aunque discerniendo entre procedimiento y habilidad ya que el procedimiento es la acción o tarea que se debe realizar para lograr un objetivo o fin en el cual la matemática está involucrada; en tanto que una habilidad matemática es la facultad personal de efectuar el procedimiento eficientemente (Williner, 2014, p. 104).

Las habilidades asociadas a la taxonomía de procedimientos permiten expresar las acciones del estudiante en las diferentes tareas matemáticas que realiza en la prueba diagnóstica:

- Habilidades de tipo aritmético: facultades para usar los números reales y las operaciones básicas y superiores, las diferentes notaciones de los números reales y establecer relaciones para decidir sobre su uso en una situación dada; establecer relaciones entre números naturales y utilizar sus propiedades para representar el cambio y la variación de un fenómeno.

- Habilidades de tipo métrico: capacidades para emplear correctamente los aparatos de medida más comunes de las magnitudes, longitud, tiempo, amplitud, capacidad, peso y superficie; utilizar el sistema métrico decimal apropiadamente para establecer relaciones entre unidades y efectuar conversiones para representar el cambio y la variación de una situación dada.

- Habilidades de tipo geométrico: facultades para construir un modelo de un concepto geométrico, para manipularlo o para hacer una representación del mismo en el plano; empleo correcto de determinados convenios para expresar relaciones entre conceptos geométricos que subyacen en fenómenos variacionales; realizar representaciones en el plano; emplear un procedimiento gráfico: expresar una imagen visual de un concepto o relación variacional; modelar fenómenos periódicos del mundo real usando relaciones y funciones trigonométricas. 
- Habilidades de tipo analítico: la capacidad para determinar las variables de una situación, establecer correctamente la interdependencia de las magnitudes variables, representar situaciones de cambio a través de ecuaciones, gráficas y tablas; traducir entre una y otra de las distintas representaciones de una función, relacionar expresiones algebraicas y gráficas empleando sus propiedades y determinar procesos infinitos implícitos en las notaciones decimales.

A la elaboración, comparación y ejercitación de procedimientos descritos en este apartado se le conoce como proceso ECEP. Se asume que un estudiante ha desarrollado su pensamiento variacional si tiene la capacidad de resolver situaciones de variación y cambio esgrimiendo algunas de las habilidades asociadas al proceso ECEP; cuando en el análisis de las respuestas de los estudiantes no es posible evidenciar dicha capacidad, se hablará de dificultad asociada al pensamiento variacional.

\section{ASPECTOS METODOLÓGICOS}

En este estudio se usó una metodología cualitativa. Para caracterizar las dificultades emergentes de la resolución de problemas variacionales se diseñó una prueba diagnóstica (Fiallo y Parada, 2014) formada por trece problemas de opción múltiple con cinco posibles soluciones, donde sólo una es la correcta. Al realizar la prueba, el estudiante debía elaborar un documento adicional llamado "hoja de procesos", explicando cómo llegó a la solución seleccionada. Los problemas que conformaron esta prueba fueron elegidos de una serie de situaciones, que han sido elaboradas por el Grupo de Investigación en Educación Matemática de la UIS para las pruebas de caracterización del pensamiento variacional de los estudiantes de nuevo ingreso a la universidad. Con el objetivo de analizar la pertinencia de los problemas propuestos en la prueba diagnóstica, particularmente en lo relacionado con el uso de procedimientos aritméticos, geométricos, métricos o analíticos, se hizo un estudio exploratorio con estudiantes de segundo semestre del la UIS. A partir de este estudio se eligieron diez de los trece problemas iniciales, considerados idóneos para caracterizar las dificultades de los estudiantes y para definir procedimientos susceptibles de aparecer en la resolución de cada problema. La prueba diagnóstica resultante fue aplicada a 113 estudiantes de nuevo ingreso, a la Licenciatura en Matemáticas y a la Ingeniería en Sistemas, cuyo análisis permitió la caracterización de diferentes dificultades. 
Con el fin de mostrar de manera más profunda la caracterización realizada, se presenta únicamente el problema de la pelota, ya que en su solución se privilegian procedimientos aritméticos, geométricos y analíticos.

\section{EL PROBLEMA DE LA PELOTA}

El problema de la pelota es el siguiente:

Una pelota de tenis se lanza hacia arriba de modo que alcanza una altura de $5 \mathrm{~m}$ desde el piso, y se deja rebotar hasta que queda en reposo. Supóngase que en cada rebote sube hasta la mitad de la altura máxima anterior. La distancia total (aproximada) que recorre la pelota antes de quedar en reposo es:
a) $10 \mathrm{~m}$
b) $9,99 \mathrm{~m}$
c) 20 metros
d) $19,99 \mathrm{~m}$
e) No sabe

La resolución de este problema exige el uso del pensamiento variacional para identificar la variación de la distancia recorrida por la pelota a medida que avanza el tiempo. A continuación, se precisan distintos procedimientos asociados al pensamiento variacional mediante los cuales se podría resolver el problema (Barajas, 2015, p. 66):

1. Emplear números reales y sus propiedades para representar el cambio y la variación del fenómeno (habilidad de tipo aritmético), identificar una regularidad en los datos recolectados, interpretar y representar la regularidad existente con una sumatoria (tal como se muestra en la Figura 1), establecer el término general de la sumatoria; aplicar y dominar propiedades de la sumatoria (habilidades de tipo analítico).

$$
\begin{gathered}
d=10+\frac{10}{2}+\frac{10}{4}+\frac{10}{8}+\cdots \\
d=10+\frac{10}{2}+\frac{10}{2^{2}}+\frac{10}{2^{3}}+\cdots \\
d=\sum_{\substack{i=0 \\
i}} 10\left(\frac{1}{2}\right)^{i} \\
d=\frac{10}{1-\frac{1}{2}}=20
\end{gathered}
$$

Figura 1. Sumatoria prevista como solución del problema. 
2. Recurrir a una representación gráfica para representar la relación variacional entre distancia recorrida por la pelota y el tiempo (habilidad de tipo geométrico). Utilizar una sucesión para representar el cambio y expresar la distancia total como la suma de los términos; concluir, al conectar lo gráfico y lo numérico, que la distancia es 20 metros pues, lógicamente, se re-construye la distancia del primer lanzamiento de la pelota (esto refleja habilidades de tipo analítico). Los procedimientos esperados aquí se muestran en la Figura 2.

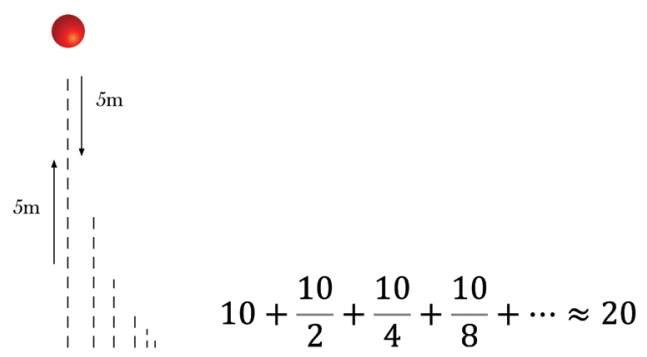

Figura 2. Procedimiento numérico esperado en la solución del problema.

3. Determinar las variables de la situación. Establecer correctamente la interdependencia de las magnitudes variables. Elaborar una tabla (como la de la Figura 3) para explorar los cambios que se producen entre las variables. Analizar la convergencia de la sumatoria: habilidades de procedimientos analíticos.

\begin{tabular}{|c|c|c|}
\hline Lanzamiento sube y baja & Distancia & Distancia acumulada \\
\hline 1 & 10 & 10 \\
\hline 2 & 5 & $10+5=15$ \\
\hline 3 & 2,5 & $10+5+2,5=17,5$ \\
\hline 4 & 1,25 & $10+5+2,5+1,25=18,75$ \\
\hline 5 & 0,625 & $10+5+2,5+1,25+0,625=19,375$ \\
\hline 6 & 0,3125 & $19,375+0,3125=19,6875$ \\
\hline$\ldots$ & Tiende a 0 & $\approx 20$ \\
\hline
\end{tabular}

Figura 3. Posible procedimiento tabular usado para resolver el problema.

Los anteriores procedimientos fueron contemplados en el análisis a priori del problema. No obstante, al revisar las hojas de procesos de los estudiantes, 
encontramos que algunos emplearon habilidades asociadas a los procedimientos métricos, al:

4. Desarrollar referentes de medida para hacer comparaciones y estimaciones, identificar relaciones entre distintas unidades utilizadas para medir cantidades de la misma magnitud y tomar una cierta cantidad como referente para determinar cuántas veces contiene una cantidad dada.

Estos procedimientos, en el orden aquí presentando, se ampliaran en la siguiente sección, a la luz de los procedimientos elaborados y ejecutados por los estudiantes que realizaron la prueba diagnóstica del curso de precálculo de la UIS.

\section{CARACTERIZANDO DIFICULTADES}

La caracterización de las dificultades se presentan considerando los cuatro tipos de procedimientos, ya que éstos permiten describir las habilidades asociadas al proceso ECEP que tienen los estudiantes para resolver problemas variacionales.

\subsection{DifiCULTADES ASOCIADAS A PROCEDIMIENTOS ARITMÉTICOS}

El problema, al ser resuelto, requiere emplear números racionales en su forma decimal o fraccionaria; 90,2\% de los estudiantes empleó decimales. Para obtener cada nueva altura se ejecuta el algoritmo de la división; se esperaría que, en estudiantes de nuevo ingreso a la universidad, las dificultades para ejecutar el algoritmo fueran mínimas (incluso, nulas). No obstante, $47 \%$ de los procedimientos asociados al cálculo de las alturas fue incorrecto.

En la Figura 4, la flecha roja señala el error en la ejecución del algoritmo de la división para calcular la altura de la pelota a partir del tercer rebote: para el tercero no es 1,75 sino 1,25 metros, y para el cuarto rebote la altura no es 0,87 sino 0,625 metros, y así sucesivamente. 


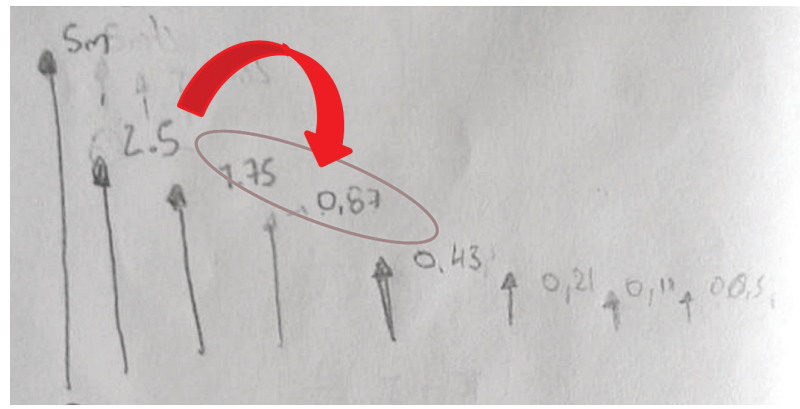

Figura 4. Ejecución errada del algoritmo de la división.

El procedimiento deja ver que el estudiante sólo se interesa en que las nuevas alturas sean cada vez más pequeñas sin tomar en cuenta todas las condiciones del problema (cada nueva altura es la mitad de la anterior). Artigue (1990) señala que una concepción heredada de los números naturales es que, al dividir un número entre otro, se obtiene un número más pequeño; por lo que el hecho de que cada medida nueva cumpla con ser menor que el anterior sirvió de control para elegir como respuesta 9,99 metros. Sin embargo, la regla "la división produce números más pequeños" no fue un elemento de control siempre: en la solución de la Figura 5, el estudiante llegó a la respuesta correcta del problema incurriendo nuevamente en errores al dividir, pues obtuvo 12,5 como resultado del cociente 1,25:2, que es significativamente más grande que 2,5.

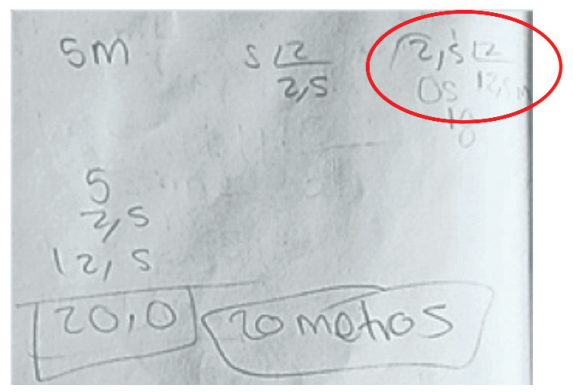

Figura 5. Procedimiento erróneo con decimales.

Esto llama la atención sobre la capacidad de los estudiantes para monitorear sus procedimientos, no se percatan de que la altura obtenida no es "más 
pequeña" que la anterior, como indican las condiciones del problema. Una posible explicación podría ser el uso indebido de la calculadora, que puede contribuir a la inhabilidad para realizar cálculos mentales o a lápiz y papel.

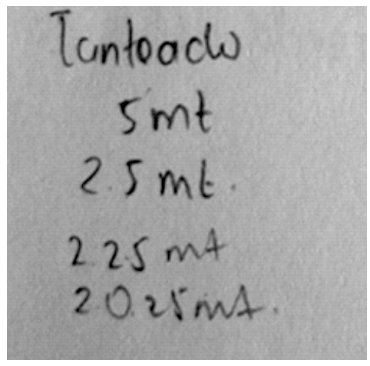

Figura 6. Ejecución errónea del algoritmo de división.

Otro procedimiento para estimar algunas alturas de la pelota es el ilustrado en la Figura 6. Se observa la estrategia de emplear propiedades de los números reales para dividir decimales, en particular la descomposición aditiva de los números decimales: tomando la segunda altura, el estudiante descompuso $2,5=2+0,5$ para dividir el 0,5 y obtener $2+0,25=2,25$ que corresponde al tercer dato.

En la Figura 6, el estudiante dejó inmodificable la parte entera del decimal para dividir solamente la parte decimal del número: de 2,5 dividió el cinco decimal obteniendo 2,5 y "juntarlo" con el número entero dos y obtener 2,25. Para el siguiente dato "desapareció" el dos decimal, y en su lugar escribió el cero para después poner los dígitos del resultado de dividir la última cifra del dato anterior entre dos: de 2,25 obtuvo 2,025. Este procedimiento erróneo permite rescatar que el cálculo mental requiere de la habilidad de poner en juego relaciones y propiedades entre los números.

Otro algoritmo que emergió para calcular la distancia total recorrida por la pelota fue el de la multiplicación.

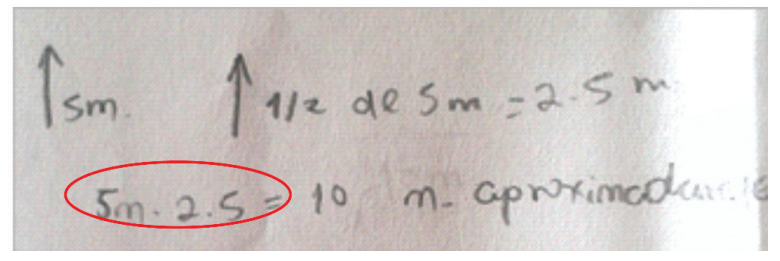

Figura 7. Dificultades en el uso del algoritmo de la multiplicación. 
El estudiante calculó 5 × 2,5 después de hallar correctamente la mitad de cinco; el procedimiento elaborado en la Figura 7 respecto al producto $5 \times 2,5$ podría interpretarse que fue visto como $5(2+0,5)$ y al aplicarle la propiedad distributiva, el estudiante multiplicó 5 por 2 y obvió el producto con el decimal (por ello el "aproximadamente"), lo que le sirve para asociar el resultado a una de las respuestas de selección múltiple del problema.

\subsection{DifiCULTADES ASOCIADAS A PROCEDIMIENTOS GEOMÉTRICOS}

En el problema, la variación implica distinguir cómo se relacionan las magnitudes, medir y analizar cómo éstas cambian; las hojas de procesos muestran la dificultad de los estudiantes para comprender el fenómeno y considerar sus características.

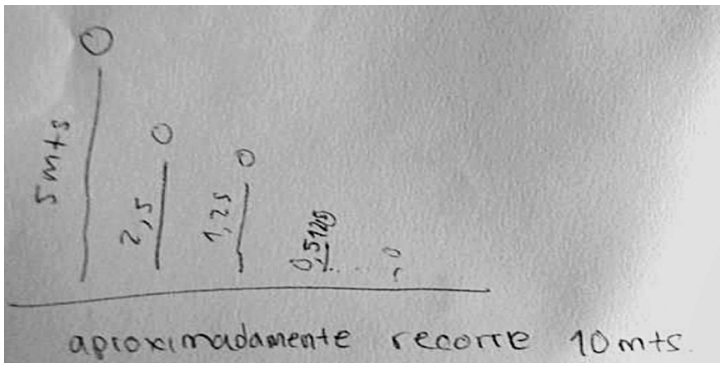

Figura 8. Representación geométrica del problema de la pelota.

El fenómeno del rebote de la pelota fue representado de diferentes maneras por, aproximadamente, 38,9\% de los estudiantes; la mitad de ellos (19) hizo una representación similar a la Figura 8, lo que indica que comprendieron erróneamente el fenómeno. Al cambiar la altura por cada nuevo rebote cuantificaron la variación de la altura, no la distancia recorrida, como lo hizo el estudiante que realizó la representación que aparece en la Figura 9. Una sencilla explicación a esto podría ser que cuando se trabaja un problema de matemáticas y se genera un modelo, se tiene tendencia a simplificar la representación, quizá por ello los estudiantes sólo se enfocaron en las alturas e ignoraron la distancia recorrida. 

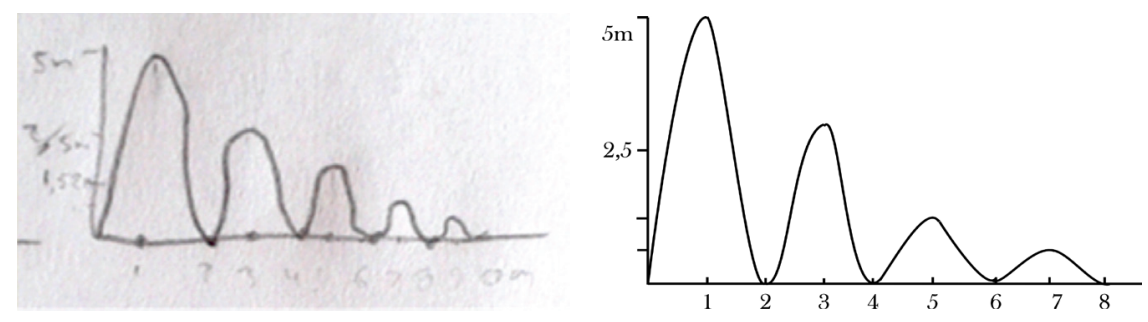

Figura 9. Representación significativa de la variación de la distancia.

En la Figura 9 (única por sus características) se explicita el plano cartesiano que subyace a la representación gráfica del fenómeno; allí el estudiante señaló la escala del eje $x$ para el número del rebote, empleando puntos que representan cada golpe de la pelota en el piso, al mismo tiempo trató de cuantificar el eje y con las alturas de la pelota, siendo el punto máximo cinco metros.

La anterior es una representación significativa, pocos estudiantes emplearon su capacidad para construir una imagen geométrica del problema que representará la relación funcional entre el número de rebotes de la pelota y la distancia recorrida por la misma (cuestión que podría ser comprensible dadas las dificultades de los estudiantes para interpretar correctamente el fenómeno de variación).

\subsection{DifiCULTADES ASOCIADAS A LOS PROCEDIMIENTOS ANALÍTICOS}

El problema de la pelota puso en jaque a los estudiantes al momento de analizar la situación para representarla, pues debían interpretar correctamente la variación del fenómeno físico: establecer que por cada acción hay una reacción igual y opuesta.

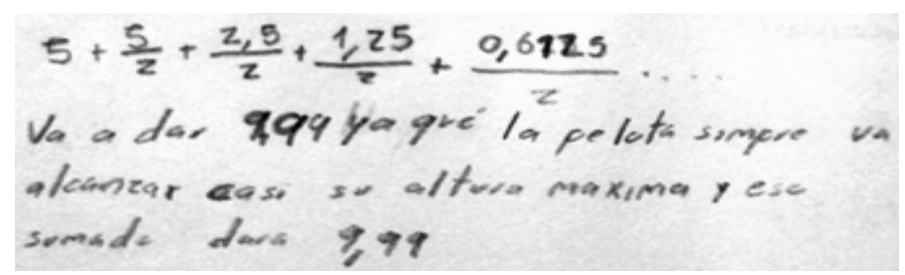

Figura 10. Simbolización de una relación funcional. 
La variación implica distinguir cómo se relacionan las magnitudes en el problema particular, empleando un sistema numérico de representación adecuado para analizar cómo éstas cambian. Por ejemplo, observemos en la Figura 10 que el estudiante usó las fracciones para registrar numéricamente la mitad de la altura en cada nuevo repique, por ello dejó invariante el denominador de cada fracción, y concluyó, en términos de la altura máxima, que ésta sería 9,99 metros justificando el resultado con la expresión "... la pelota siempre va alcanzar casi su altura máxima....

Con esta representación numérica se esperaba que el estudiante detectara algún patrón. Sin embargo, los números decimales empleados en el numerador no le permitieron encontrar una regularidad entre los términos conocidos de la sucesión para obtener el término general. La representación de los puntos suspensivos podría asociarse a cierto tipo de noción del infinito del estudiante al usarla como expresión algebraica para representar que la suma, como un proceso numérico, es infinita.

La expresión de dos líneas y media, escrita por el estudiante, da cuenta del razonamiento sobre la tendencia de la altura máxima: no podría ser 10 metros porque siempre le faltaría a la pelota una cantidad infinitesimalmente pequeña que cubrir en su rebote. Esta percepción manifiesta un tratamiento intuitivo del infinito que viene a convertirse en obstáculo cognitivo para la resolución del problema, y que ya ha sido reportado por Hitt (2003).

El procedimiento anterior permite comparar las representaciones decimales y fraccionarias de los números racionales empleadas por los estudiantes, para destacar la dificultad para determinar si es necesario realizar cálculos o dejarlos expresados para identificar patrones y regularidades; esto señala la importancia de que usen las propiedades de los números reales para establecer relaciones entre sus subconjuntos numéricos y las propiedades y, así, cuando empleen algoritmos puedan tener el control sobre éstos. Identificar las magnitudes variables correctas del problema fue una dificultad significativa en los estudiantes, se evidenció en las representaciones pictóricas (ver Figura 11): este ejercicio de dibujar supone el empleo de convenios para expresar una imagen visual de la representación de la relación de la altura máxima con el desplazamiento de la pelota en el piso. Establecer dicha relación entre las magnitudes variables correctas fue una dificultad notoria entre quienes realizaron un dibujo de la situación porque no tuvieron en cuenta que el cambio de un rebote a otro implicaba que la altura máxima alcanzada por la pelota se recorría tanto subiendo como bajando. 

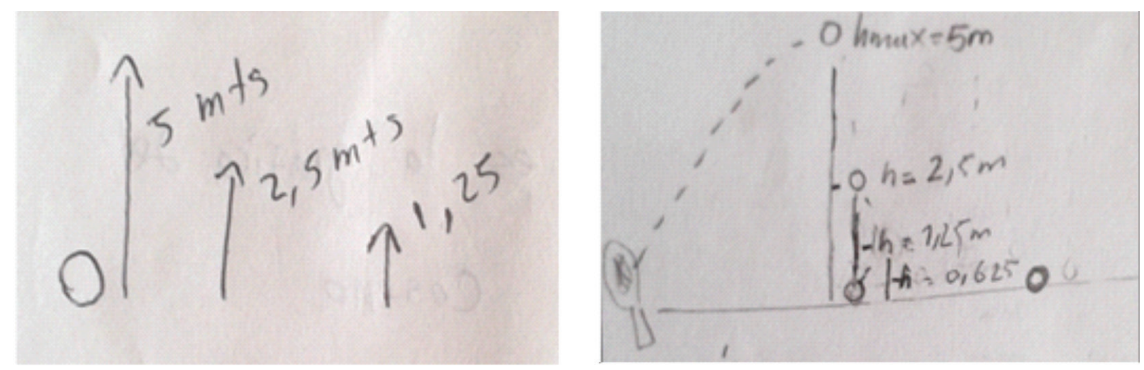

Figura 11. Representaciones pictóricas erradas del problema de la pelota.

Las evidencias de la Figura 11, y en lo expuesto desde el inicio de los hallazgos, dejan ver que los estudiantes no reconocen la interdependencia de las variables involucradas en esta situación. Esta dificultad condujo a los estudiantes a considerar los procedimientos ejecutados como correctos pese a ser erróneos, como algunos de los presentados en las categorías de los procedimientos aritméticos. Por lo tanto, las dificultades en la resolución de este problema se produjeron, principalmente, porque los estudiantes no analizaron correctamente la situación de cambio, lo cual incidió en la creación de representaciones inadecuadas de la situación.

En la Figura 12 la tabla de valores fue otra representación empleada: sólo $5,3 \%$ de los estudiantes consideró el uso de tablas para observar el cambio y la interdependencia de las magnitudes variables del problema y, a su vez, observar patrones o regularidades que les permitieran establecer procesos de generalización.

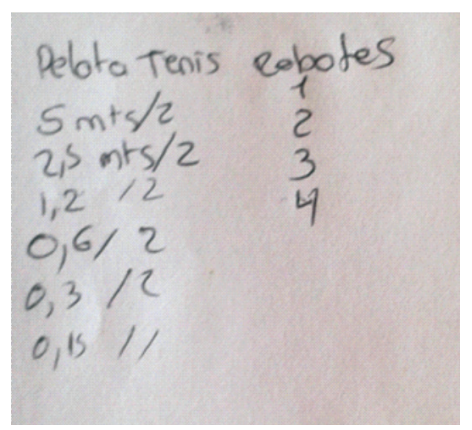

Figura 12. Elaboración de una tabla de valores. 
Del procedimiento anterior, se pueden resaltar los siguientes aspectos:

- La manera de registrar las alturas, aunque se comprende que quizá sólo intentaba expresar el cociente entre dos en cada nuevo rebote.

- La falta de claridad sobre qué hacer con la tabla de valores, lo que indica que el estudiante no reconoce la variación conjunta de las variables.

- El estudiante invirtió las columnas de las magnitudes involucradas considerando como variable independiente a la altura de la pelota, lo que indica su dificultad para establecer correctamente la interdependencia entre las magnitudes variables y para transferir los datos a otra forma de representación (ecuación).

Esto evidencia las dificultades para reconocer que una variable es una imagen abstracta de una magnitud que varía y para emplear tablas de valores que permitan establecer procesos de generalización. El procedimiento de la Figura 13, desde un análisis numérico, evidencia la elaboración de un método que lleva a una generalización: el estudiante construyó el término n-ésimo de la sucesión $2\left(\frac{5}{2^{\prime \prime}}\right)$ y la llevó a la sumatoria, pero su conocimiento conceptual no le permitió avanzar en lo procedimental para registrar una respuesta.

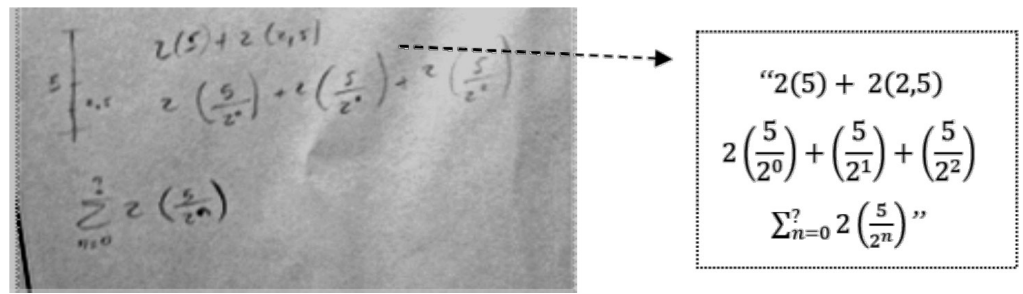

Figura 13. Generalización del proceso infinito del problema de la pelota.

En este procedimiento la generalización realizada está favorecida por el uso del sistema simbólico de las fracciones. Esta representación fue empleada en otro procedimiento. La Figura 14 muestra un mayor acercamiento a la generalización de la situación; el estudiante puso en juego sus nociones de sumatoria y límite sin éxito: 


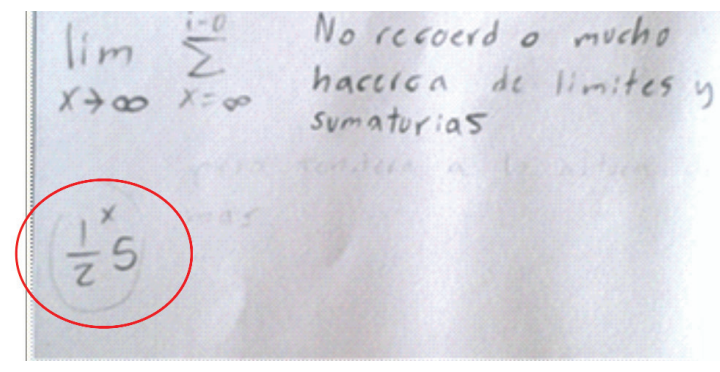

Figura 14. Procedimiento analítico con noción de sumatoria y límite.

El análisis de este caso sugiere:

1. El estudiante intentó emplear la propiedad genérica del límite.

2. Intentó emplear los recursos de sucesiones y límites vistos en el colegio que, según la evidencia, no recordó completamente como tampoco los procedimientos implicados para la solución, sino que trató de emplear la memoria para adaptarlos, ya que mezcló las notaciones de límites y sumatoria arbitrariamente.

También el estudiante:

3. No expresó correctamente el término general de la sucesión, pues no notó que $\frac{1}{2}^{x} 5=\frac{5}{2}$ y no $\frac{1}{2^{x}} 5$ como quizás lo razonaba.

4. Tiene dificultades con el tema, insinuando que la respuesta es incompleta o incorrecta.

De los procedimientos de las Figuras 13 y 14, se puede interpretar que los estudiantes emplean variables en una relación funcional identificando que la variación de una magnitud afecta a la otra independientemente de cómo se proporcione la información (Ursini y Trigueros, 2006). Otro uso dado a la variable lo observamos en la respuesta de otro estudiante. La Figura 15 muestra que se trató como una incógnita específica al reconocer la existencia de algo desconocido que se puede determinar. 


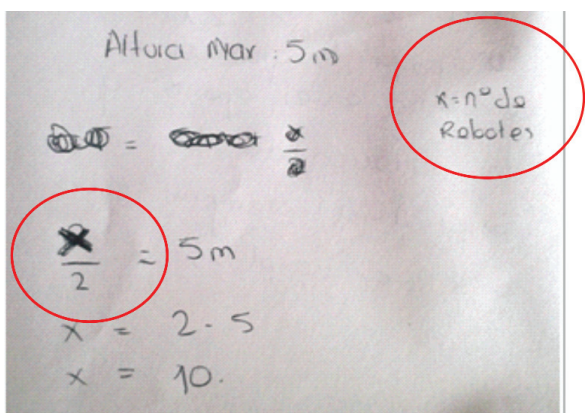

Figura 15. Uso de la variable como incógnita.

En la Figura 15, el estudiante simbolizó la cantidad desconocida y la utilizó para plantear la ecuación; determinó correctamente la cantidad desconocida que aparece en la ecuación y realizó las operaciones algebraicas pertinentes para despejar. Los óvalos dan cuenta de que para el estudiante fue ambiguo lo que representaba la variable equis pues en la parte superior derecha estableció que es el número de rebotes, pero al montar la ecuación vendría siendo la altura de la pelota pues ésta está relacionada con "la mitad".

Ursini y Trigueros (2006) han reportado esta problemática relacionada con los usos de la variable: las autoras comprobaron que, en los cursos de álgebra elemental, aparecen esencialmente tres usos (o niveles de abstracción) de la variable: como incógnita específica, como número general, y las variables en relación funcional.

El tercer uso de la variable fue revelado por un estudiante quien puso en juego sus "nociones formales" de cálculo diferencial en la ejecución del procedimiento.

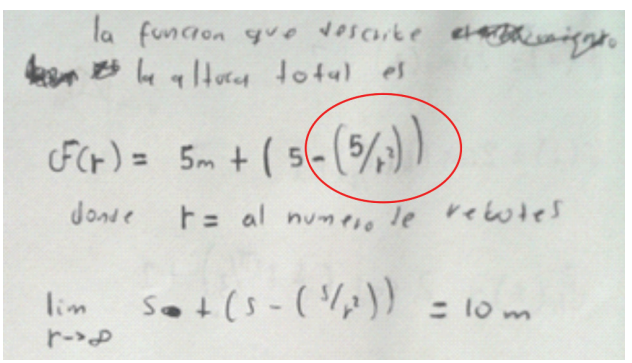

Figura 16. La variable simbolizando una relación funcional. 
Los procedimientos en la Figura 16 muestran que el estudiante obtuvo una función $f: N \rightarrow R$ para simbolizar la altura de la pelota en función del número del rebote. Al revisar el procedimiento podemos decir que:

- f(r) no modela bien el fenómeno porque el estudiante no analizó correctamente la situación de cambio (la pelota, en su caso, sólo bajó o subió); además, al revisar la función para casos particulares, falla significativamente:

$$
\begin{aligned}
& f(0)=5+\left(5-\left(\frac{5}{0^{2}}\right)\right) \text { no está definida; } \\
& f(1)=5+\left(5-\left(\frac{5}{1^{2}}\right)\right)=5+0=5, f(2)=5+\left(5-\left(\frac{5}{2^{2}}\right)\right)=5+\frac{15}{4}=\frac{35}{4}, \\
& f(3)=5+\left(5-\left(\frac{5}{3^{2}}\right)\right)=5+\frac{40}{9}=\frac{85}{9}, \ldots
\end{aligned}
$$

- El estudiante tuvo dificultad para expresar acertadamente que con cada nuevo rebote, la pelota alcanza la mitad de la altura anterior. Esto podría deberse a una dificultad en el uso del lenguaje algebraico pues confundió $5 / 2^{r} \operatorname{con} 5 / r^{2}$.

Pese al error el procedimiento resulta interesante porque:

- Se intentó modelar el cambio de la nueva altura,

- Se intentó capturar la tendencia de la distancia total cuando el número de rebotes tienden a infinito, aproximando la convergencia de la serie con el límite cuando $n$ tiende a infinito de $f(r)$.

\subsection{DifiCUltadeS ASOCIADAS A PROCEDIMIENTOS MÉTRICOS}

La elaboración de procedimientos para solucionar los problemas de la prueba diagnóstica no exigía procedimientos métricos. No obstante, 46,9\% de los estudiantes realizó algún procedimiento con el sistema métrico decimal; no se contempló que los estudiantes convirtieran unidades en el problema de la pelota. En la Figura 17 realizamos la conversión correcta de metros a centímetros para los nueve datos considerados por el estudiante, y trascribimos el registro del estudiante. 


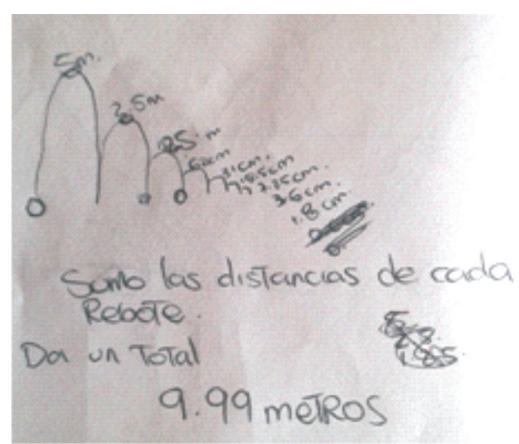

\begin{tabular}{|c|c|c|}
\hline \multicolumn{3}{|c|}{ CONVERSIÓN } \\
\hline CORRECTA & & Del \\
\hline Metros & a Centímetros & Estudiante \\
\hline 5 & $=500$ & $5 m$ \\
\hline 2,5 & $=250$ & $2,5 \mathrm{~m}$ \\
\hline 1,25 & $=125$ & $1,25 m$ \\
\hline 6,25 & $=62,5$ & $62 \mathrm{~cm}$ \\
\hline 0,3125 & $=31,25$ & $31 \mathrm{~cm}$ \\
\hline 0,15625 & $=15,625$ & $15 \mathrm{~cm}$ \\
\hline 0,078125 & $=7,8125$ & $7,75 \mathrm{~cm}$ \\
\hline 0,3980625 & $=3,980625$ & $3,6 \mathrm{~cm}$ \\
\hline 0,01953125 & $=1,953125$ & $1,8 \mathrm{~cm}$ \\
\hline
\end{tabular}

Figura 17. Contrastando acciones de conversión.

El estudiante intentó seleccionar unidades de medida apropiadas para cada nueva medición de la altura; destaca la conversión de unidades que realizó, trabajando conjuntamente metros y centímetros. El estudiante intentó planificar y monitorear el progreso de su solución, aunque no es claro si los procedimientos elaborados condujeron a la opción elegida como respuesta (9,99 metros) pues al sumar los tres primeros datos obtendría $8,75 \mathrm{~m}$. Podríamos decir que este estudiante evidencia facultades para identificar relaciones entre distintas unidades utilizadas para medir cantidades de la misma magnitud (MEN, 2006).
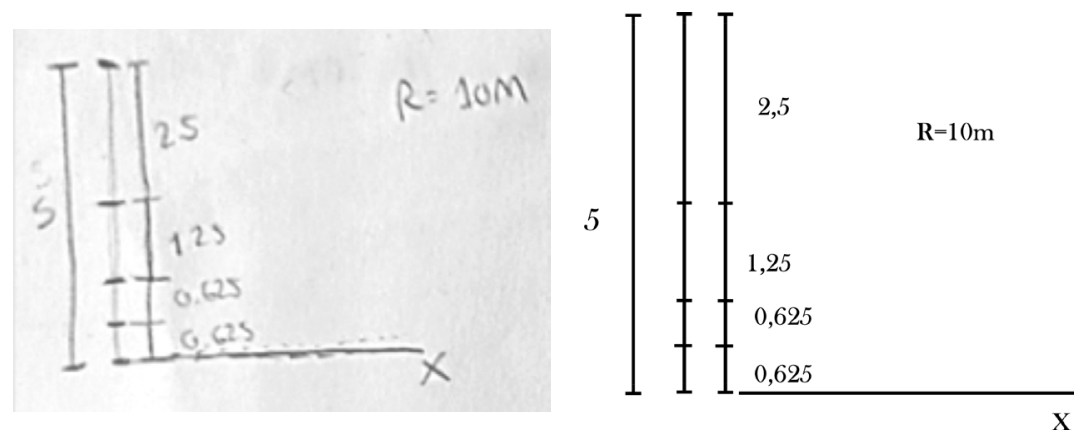

Figura 18. Sumando cantidades de longitud.

Finalmente, el procedimiento presentado en la Figura 18 destacó entre todos por no ser rutinario, elaborado desde el cálculo mental de sumar cantidades de 
longitud las cuales se pueden comparar entre sí: la comparación se ve facilitada si se toma una cierta cantidad [u] como referente y se determina cuántas veces contiene una cantidad dada [a] a [u] (Godino, Batanero y Roa, 2002).

Aquí interpretamos que el estudiante, después del primer rebote, acumuló, en un segmento, las alturas para los siguientes cuatro rebotes; señaló cada rebote con una marca en el segmento, de manera que completó otro segmento de igual longitud al que representa la máxima altura del primer rebote concluyendo que la distancia total es $10 \mathrm{~m}$.

Aunque el procedimiento señala nociones de tendencia y aproximación en el proceso infinito de acumular segmentos, el análisis realizado por el estudiante del fenómeno de cambio de la pelota no fue correcto pues consideró la altura de la pelota lo cual invalida el procedimiento; sin embargo, destaca su habilidad para desarrollar referentes de medida para hacer comparaciones y estimaciones (NCMT, 2003) aunque no empleó otro tipo de procedimiento para evaluar su resultado.

\section{CONCLUSIONES}

Los hallazgos expuestos a partir del problema de la pelota permiten una reflexión sobre las dificultades en el pensamiento variacional que experimentan estudiantes de nuevo ingreso desde el dominio conceptual y procedimental de los números reales, aun cuando su uso se realiza desde temprana edad escolar; convendría en algún momento de su formación fundamentarlo a partir de una construcción formal.

Las descripciones de las habilidades de cada tipo de procedimiento permitieron una caracterización de las dificultades a las cuales se enfrentaron los estudiantes del curso de precálculo de la UIS y que se explicaron en la solución del problema de la pelota. A continuación, se presentan las categorías de dificultades que establecimos en relación al análisis global a este problema y a los otros que conformaban la prueba y que permiten elucidar dichas categorías; además, se esbozan algunas tareas matemáticas que podrían ayudar a los estudiantes a superar estas dificultades.

\subsection{DifiCUltades EMERGENTES A LOS PROCEDIMIENTOS DE TIPO ARITMÉtICO}

Se manifestaron al utilizar propiedades y relaciones numéricas para realizar cálculos, ya sea escritos o mentales. La solución al problema pone de manifiesto 
la necesidad de emplear correctamente diferentes representaciones para escribir los números y reconocer el efecto de una representación u otra en la resolución del problema. Por lo anterior, sería pertinente implementar tareas matemáticas que sean abordadas con distintos tipos de notación y discutir con los estudiantes lo que aporta una u otra opción. Por ejemplo, para el caso del problema analizado, una notación en fracciones, correctamente utilizada, facilitaría la representación del término n-ésimo.

\subsection{DifiCULTADES EMERGENTES A LOS PROCEDIMIENTOS DE TIPO GEOMÉTRICO}

Realizar representaciones geométricas y usarlas para resolver problemas en diferentes contextos. Ante esta dificultad, el profesor podría contrastar, en la clase de matemáticas, las diferentes representaciones geométricas que proponen los estudiantes y debatir con el grupo los pros y los contras de una u otra representación.

\subsection{DiFICULTADES EMERGENTES A LOS PROCEDIMIENTOS DE TIPO ANALÍTICO}

Las principales dificultades de los estudiantes al resolver el problema de la pelota fueron: el tipo de interpretación que se le asignó, el grado de profundidad con que se reflexionó, así como la estrategia de solución a plantear. Otras estuvieron asociadas a:

- Reconocer y describir regularidades y patrones en distintos contextos.

- Distinguir cómo se relacionan las magnitudes en el problema particular y reflexionar lo variante e invariante del problema.

- Establecer correctamente la interdependencia entre las magnitudes variables para transferir los datos a otra forma de representación.

- Emplear tablas de valores para observar patrones o regularidades que permitan establecer procesos de generalización.

- Generalizar los resultados de operaciones aritméticas y manipular operaciones algebraicas.

- Reconocer que una variable es una imagen abstracta de una magnitud que varía. 
- Reconocer la variación conjunta de las variables involucradas en una relación funcional, independientemente de la representación utilizada.

- Establecer correctamente la interdependencia entre las magnitudes variables para transferir los datos a otra forma de representación.

- Simbolizar una relación funcional apoyándose en el análisis de los datos del problema.

- Analizar procesos infinitos que subyacen en las notaciones decimales.

- Identificar en qué se parecen y diferencian los términos de una sucesión.

- Encontrar el término n-ésimo de una sucesión.

- Emplear métodos numéricos y analíticos para analizar la convergencia de una serie.

- Reconocer características de los procesos infinitos utilizando diversas representaciones o explicaciones verbales.

- Utilizar tablas, expresiones orales, expresiones algebraicas, ecuaciones y gráficas y hacer traducciones entre estas representaciones para analizar funciones.

- Utilizar aproximaciones numéricas o gráficas de la función para deducir intuitivamente el límite de una función.

Para mejorar el desempeño de los estudiantes, en relación a los procedimientos analíticos, conviene involucrarlos en la resolución de más problemas como el de la pelota, ayudándoles a notar todo lo que varía en el fenómeno que se involucre. Aunque en algunas soluciones se manifestaron intentos de modelar, se evidenció falta de recursos para ello (o huecos en su conocimiento matemático), por ejemplo, un adecuado uso de la notación de fracciones y la determinación del término n-ésimo.

\subsection{DIFICULTADES EMERGENTES A LOS PROCEDIMIENTOS DE TIPO MÉTRICO}

Las dificultades asociadas a las habilidades de tipo métrico no son, seguramente, nuevas para los profesores de matemáticas quienes con frecuencia observan que los estudiantes usan, con alguna dificultad, relaciones entre distintas unidades utilizadas para medir cantidades de la misma magnitud, realizan operaciones entre magnitudes, sus propiedades y las relaciones entre ellas para representar y analizar procesos infinitos, así como desarrollar apropiadamente referentes de medida para hacer comparaciones y estimaciones. Para contribuir a subsanar estas dificultades 
es apropiado proponer con mayor frecuencia tareas matemáticas que involucren distintas unidades de medida en un mismo problema.

Para finalizar, no es de sorprender el bajo desempeño de los estudiantes de nuevo ingreso en el curso de Cálculo Diferencial el cual requiere dominio de las habilidades emergentes del proceso ECEP asociadas al pensamiento variacional. Estas habilidades demuestran la competencia matemática del estudiante frente a situaciones de variación y cambio. Los conceptos y procedimientos que los estudiantes han adquirido en el colegio requieren fortalecerse para restablecer la conexión entre ellos de manera que se favorezcan su competencia matemática.

En este sentido las dificultades estudiadas y alcanzadas en este trabajo muestran oportunidades de intervención. Pensar en cómo concretar esas acciones y cómo, incluso, llevar estos resultados a la educación básica y media ha sido y sigue siendo una tarea de la comunidad investigadora de la educación matemática y de los profesores que imparten matemáticas. Al divulgar los resultados de esta investigación, se quiere contribuir en la sensibilización de los profesores de matemáticas sobre la importancia de promover el desarrollo del pensamiento variacional a partir de, entre otras actividades matemáticas, problemas que permitan a los estudiantes usar el conocimiento conceptual de este pensamiento y los procesos matemáticos que contribuyen a su desarrollo, tomando como referencia las dificultades asociadas al proceso de elaboración, comparación y ejecución de procedimientos presentadas aquí.

\section{REFERENCIAS}

Abrate, R., Pochulu, M. y Vargas, J. (2006). Errores y dificultades en matemática. Análisis de causas y sugerencias de trabajo. Buenos Aires: Universidad Nacional de Villa María. Recuperado de http://unvm.galeon.com/Libro1.pdf

Artigue, M. (1990). Epistémologie et didactique. Recherches en Didactique des Mathématiques, 10(2/3), 241-286.

Artigue, M. (1995). La enseñanza de los principios del Cálculo: problemas epistemológicos, cognitivos y didácticos. En M. Artigue, R. Douady, L. Moreno y P. Gómez (Eds.), Ingeniería didáctica en educación matemática (pp. 97-140). México: Grupo Editorial Iberoamérica.

Barajas, C. (2015). Elaboración, comparación y ejercitación de procedimientos: una mirada desde la resolución de problemas que implican fenómenos de variación. Tesis de maestría no publicada. México: CICATA-IPN. 
Botello, C. (2013). Procesos de Seguimiento y Acompañamiento Académico a Estudiantes de Cálculo Diferencial: Un Aula Experimental para Profesores de Matemáticas en Formación (Tesis de maestría no publicada). Colombia: Universidad Industrial de Santander.

Cadenas, R. (2007). Carencias, dificultades y errores en los conocimientos matemáticos en alumnos del primer semestre de la escuela de educación de la Universidad de los Andes. Revista Científica Ciencias Humanas (Orbis), 2(6), 68-84. Recuperado de http://www.redalyc.org/pdf/709/70920605.pdf

Díaz, J. (2009). Los estudiantes de Cálculo a través de los errores algebraicos. México: CINVESTAV. Recuperado el 15 de mayo de 2014 de http://mattec.matedu.cinvestav. mx/el_calculo/data/docs/GZe5a1110t9.pdf

Fiallo, J. E. y Parada, S.E. (2014). Curso de pre-cálculo apoyado en el uso de geogebra para el desarrollo del pensamiento variacional. Revista Científica. 20.

Godino, J., Batanero, C., y Roa, R. (2002). Medida de magnitudes y su didáctica para maestros. En Godino, J. (Ed.), Didáctica de las matemáticas para. Departamento de Didáctica de la Matemática. Universidad de Granada, pp. 615-633.

Hitt, F. (2003). Dificultades en el aprendizaje del cálculo. XI Encuentro de Profesores de Matemáticas del Nivel Medio Superior. Morelia: Universidad Michoacana de San Nicolás de Hidalgo.

Ministerio de Educación Nacional (MEN). (1998). Lineamientos curriculares en matemáticas. Bogotá: Autor.

Ministerio de Educación Nacional (MEN). (2006). Estándares básicos de competencias en Matemáticas. Bogota: Autor. [Versión en línea]. Recuperado de http://www.mineducacion.gov.co/1621/articles-116042_archivo_pdf2.pdf

NCTM. (2003). Principios y Estándares para la Educación Matemática. Traducción de M. Fernández (Traducción de la versión del 200 del NCTM). SAEM Thales. Sevilla.

Parada, S. (2012). Una estructura curricular para atender la problemática relacionada con el curso de Cálculo I en la Universidad Industrial de Santander. Documento interno no publicado de la Escuela de Matemáticas. Bucaramanga, Colombia: UIS.

Rico, L. (1995). Errores y dificultades en el aprendizaje de las matemáticas. En Lilpatrick, J., Gómez, P. y Rico, L. (Eds.), Educación Matemática. Errores y dificultades de los estudiantes. Resolución de problemas. Evaluación. Historia. (Vol. 1, pp. 69-108). México: Grupo Editorial Iberoamérica, S.A. de C.V.

Seduca. (2005). Interpretación e Implementación de los Estándares Básicos de Matemáticas. Colombia: Gobernación de Antioquia.

Ursini, S. y Trigueros, M. (2006). ¿Mejora la comprensión del concepto de variable cuando los estudiantes cursan matemáticas avanzadas? Educación Matemática, 18(3), 5-38. 
Valdivé, C. y Garbin, S. (2013). “¿Cómo piensan los estudiantes el infinitesimal antes de iniciar un curso de análisis matemático?" Paradigma, XXXIV(1), 117 - 144.

Williner, B. (2014). Habilidades matemáticas referidas el concepto de Derivada y uso de tecnología. Revista de Didáctica de las Matemáticas (Números), 8(1), 101-124.

\section{CLAudia BARAJAs ARENAS}

Dirección postal: $\quad$ Carrera 11 \#10N-36 Bucaramanga, C.P. 680002 